\title{
Wearable multi-antenna multi-band measurement system for personal radio-frequency exposure assessment
}

\author{
P. Van Torre ${ }^{1}$, S. Agneessens ${ }^{1}$, R. Aminzadeh ${ }^{2}$, A. Thielens ${ }^{2}$, M. Van den Bossche ${ }^{2}$, \\ W. Joseph ${ }^{2}$ and H. Rogier ${ }^{1}$ \\ ${ }^{1}$ Ghent University, INTEC - imec/IDlab, Ghent-Zwijnaarde, Belgium \\ 2 Ghent University, INTEC - WAVES, Ghent-Zwijnaarde, Belgium
}

\begin{abstract}
Rising concern about the potential harmfulness of human radio-frequency exposure causes scientists to be highly interested in measuring these exposure levels. The set of measured signal levels on a map of locations is then used for epidemiological research, looking for correlation to the frequency of incidence of certain illnesses. Currently available hand-held exposimeters have the disadvantage that the measured signal levels are often significantly different from the exposure to which the human body is actually subjected. Therefore, a personal distributed exposimeter was developed and documented in this paper. Multiple on-body antennas are employed in order to measure the electromagnetic field strengths directly on the body. In this design, 11 commonly used frequency bands are measured, with front and back textile antennas for each of those bands. These 22 antennas are implemented in substrateintegrated-waveguide technology, thereby combining a compact size with a large bandwidth. The measurement system operates autonomously, through measurement, control and datalogging circuitry directly integrated onto the antennas. The textile-antenna based nodes are unobtrusively integrated into a garment, resulting in maximum comfort for the user. A measurement campaign using the system is currently underway in a number of European countries, yielding a large amount of valuable and unique data for epidemiological analysis.
\end{abstract}

Keywords - body-centric, radio-frequency exposimetry, dosimeter, textile antenna

\section{INTRODUCTION}

Nowadays, wireless technology is ubiquitous. However, rising concern about the safety of long-term exposure to radio-frequency fields exists. Therefore, it is important to perform measurement campaigns to assess the exposure of individuals in modern-day circumstances. The measurement data can then be analyzed in subsequent epidemiological research, to potentially confirm a statistically significant link to the frequency of certain illnesses. Hand-held exposimeters are available on the market, such as the EME SPY 200 by Mi- crowave Vision Group, but these devices have the disadvantage that their measurements are influenced by the position at which they are carried by the test person. Additionally, reflections and shadowing by the human body occur. Therefore, the field strength of the electromagnetic waves impinging on the antenna of the hand-held device is often very different from the signal level actually experienced by the human body. Because of this, hand-held devices generally underestimate the exposure [1].

For the above reasons, a distributed body-worn exposimeter was proposed and developed, inherently allowing a more accurate measurement of human exposure to radio-frequency energy. On-body textile antennas are now used to capture the signal actually impinging on the body. Multiple antennas can be worn on the body, collecting measurements of waves from many different angles. Shadowing of the signals by the human body, as well as small-scale fading caused by reflections on the body are countered by such a system. The measurement protocol to obtain more accurate data is outlined in [2], even when using only a two-antenna system, with front and back antennas.

Multiple antennas perform measurements in different frequency bands. The potential harmfulness of exposure is indeed likely to depend on the wavelength of the radiation. The human body provides ample surface for a multi-antenna platform performing synchronous measurements in multiple frequency bands.

To enable multi-band on-body exposimetry in an unobtrusive way, a distributed exposimeter was developed where every on-body active textile antenna contains its own circuitry for measurement and logging of the data. Each intelligent exposimeter node is connected to a common bus system, allowing convenient readout of the data from the different local flash memories, integrated into each node. Via the same bus system, extra features are also available, such as real-time monitoring of the operational status of the nodes, as well as placing time stamps for synchronization purposes or marking reference points in the measurement in all the nodes' flash memories. 
The paper is organized as follows. In Section II, the construction of the antennas and the circuitry integrated onto it are presented, as well as the master node controlling the full system. Section III displays measurement results to illustrate the correct operation of the distributed exposimeter. Finally, the conclusions are outlined in Section IV.

\section{MATERIALS AND METHODS}

Each exposimeter node is composed of a flexible textile substrate-integrated-waveguide (SIW) antenna, with the electronic circuitry implementing its functionality integrated on its feed plane. Each node is a very compact unit, easily integrated into clothing. All on-body nodes, further called slave nodes, collaborate in a coordinated manner through a common bus system. A hand-held master node, interfaced to the bus, provides synchronization and real-time performance monitoring during measurement and data readout. A picture of an on-body node is shown in Fig. 1.

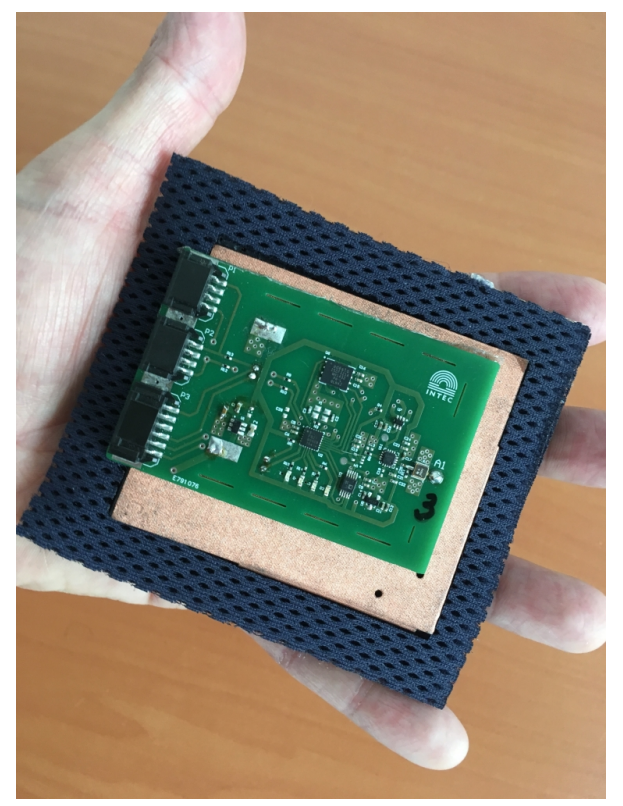

Fig. 1: Back side of on-body node for the DECT band

\section{A. Slave node textile SIW Antenna}

SIW technology leverages the design of textile antennas with robust and reliable on-body performance, unaffected by the presence of the wearer [3]. The dedicated antenna design for the exposimeter relies on SIW-related miniaturization techniques $[3,4]$ to realize compact antennas with sufficient bandwidth to cover an entire frequency band with just a single antenna. The dimensions of the largest antenna are $100 \times 100 \times 11 \mathrm{~mm}$, whereas the smallest antenna is only 90x100x6 mm. Their operating frequency ranges are shown in Table 1.

\section{B. Slave node circuit design}

The design of the slave node's circuit, which is deployed on the antenna and then into the garment of a test person, is based on a previous version of the system, documented in [5]. A logarithmic radio-frequency (RF) detector chip forms the heart of the circuit. Depending on the frequency band of interest, either an ADL5513 (up to $2.45 \mathrm{GHz}$ ) or an AD8318 (higher bands) detector chip is applied, both manufactured by Analog Devices, Inc. These detectors provide an output voltage proportional to the detected RF level, sampled by a 16-bit analog-to-digital converter (ADC) and further processed by a low-power micro controller. The latter chip also controls read and write operations to the bus and to the $32 \mathrm{Mbit}$ local flash memory.

For lower frequency bands, low-cost standard FR4 printed-circuit board (PCB) material is applied, whereas for the $5.8 \mathrm{GHz}$ band $500 \mu$ m-thick Rogers Isola I-tera highfrequency laminate, is employed. The latter substrate features lower losses at the higher frequencies.

For every different band, a suitable ceramic band pass filter is mounted on the PCB, to suppress out-of-band signals. All applied band pass filters are very small surface mounted components. Table 1 lists the filters for the 11 different bands measured by the system. For most bands, the filter range corresponds exactly to the band of interest, but for a few bands a compromise had to be made, choosing the best matching commercially available filter.

\section{Master node hardware}

The master node is a hand-held unit, allowing portable control of the measurement system. The unit contains an LCD screen and control buttons, and forms the interface between the user and the custom internal bus system. Additionally, the master node serves as an interface between the slave nodes on the bus and a PC that reads out the data from all the local flash memories of the slave nodes, through one central connection point.

At the same time, the master node monitors the correct operation of the measurement system. All slave nodes have a unique address, shown on the LCD as a character from "A" to "V", for the 22 nodes deployed in the garment. These characters only are indicated on the LCD if the nodes respond on the bus, a blank position indicates a faulty node. The characters 
Table 1: Ceramic bandpass filters

\begin{tabular}{llll}
\hline $\begin{array}{l}\text { Frequency } \\
\text { Band }\end{array}$ & $\begin{array}{l}\text { Frequency } \\
\text { Range } \\
(\mathrm{MHz})\end{array}$ & $\begin{array}{l}\text { Filter } \\
\text { Range } \\
(\mathrm{MHz})\end{array}$ & $\begin{array}{l}\text { Manufacturer and } \\
\text { Type nr. }\end{array}$ \\
\hline LTE800DL & $791-821$ & $791-821$ & GSRF TA1071A \\
GSM900UL & $876-915$ & $880-915$ & Triquint TQ856671 \\
GSM900DL & $921-960$ & $925-960$ & Triquint TQ856528 \\
GSM1800UL & $1710-1785$ & $1710-1785$ & Triquint TQ856654 \\
GSM1800DL & $1805-1880$ & $1805-1880$ & Triquint TQ855860 \\
DECT & $1880-1900$ & $1850-1910$ & Triquint TQ855849 \\
UMTS UL & $1900-1980$ & $1920-1980$ & Triquint TQ855817 \\
UMTS DL & $2110-2170$ & $2110-2170$ & EPCOS/TDK \\
& & & B39212B7750C810 \\
WiFi 2G & $2400-2485$ & $2400-2484$ & Triquint TQ855916 \\
LTE2600DL & $2620-2690$ & $2620-2690$ & GSRF TA1232A \\
WiFi 5G & $5150-5875$ & $5150-5875$ & Johanson \\
& & & Technology \\
& & & $5515 B P 15 C 725 E$ \\
\hline
\end{tabular}

are displayed in upper case for nodes operating in logging mode and lower case for nodes in standby mode.

As an extra feature, a time stamp can be set by pushing a dedicated button on the master node. The time stamp command is then broadcast over the bus and received by all slave nodes, which record the time stamp synchronously in their local flash memories. Owing to this feature, specific markers can be set during a measurement campaign, for synchronization purposes, or for marking specific points of interest in the measurement.

The on-body nodes are integrated into a jacket, allowing to wear the measurement system in a convenient way. The jacket including the system, as well as the master unit and battery pack are displayed in Fig. 2. A picture of a test person wearing the exposimeter jacket in an anechoic chamber, for calibration purposes, is shown in Fig. 3.

\section{Embedded software, slave nodes}

The embedded software controls the functionality of the system on a number of different levels. For the slave nodes, the following functions are implemented:

\section{Measuring the data}

The logarithmic radio-frequency detector circuit receives the bandpass filtered signal from the antenna. The detector

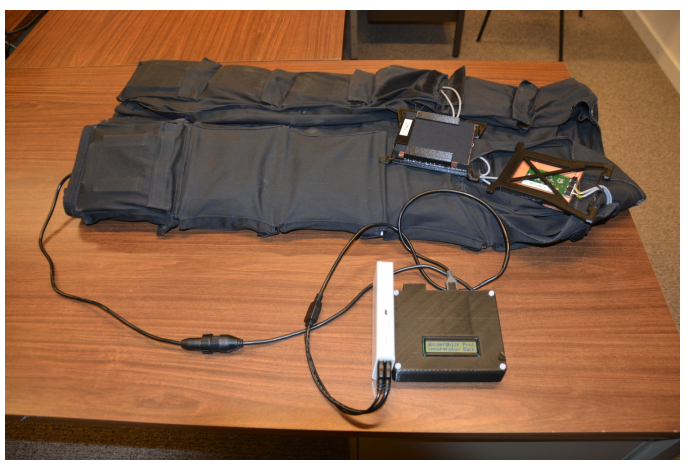

Fig. 2: System overview showing front and back side of two nodes and master unit. The remaining 20 nodes are integrated into the jacket.

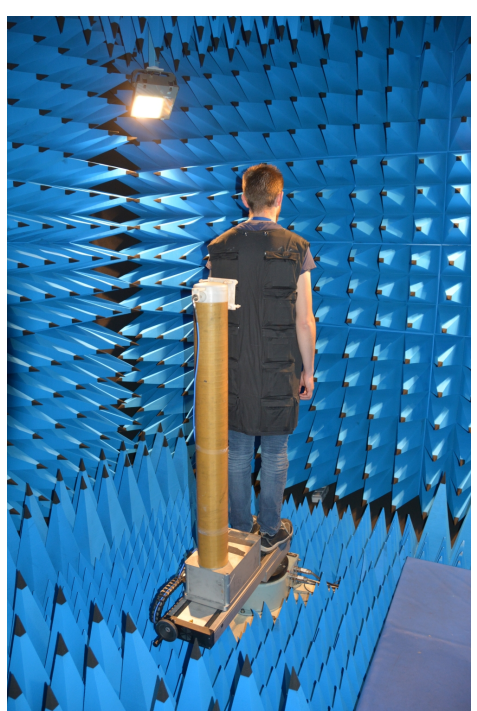

Fig. 3: Test person wearing the personal distributed exposimeter jacket, in the anechoic chamber for calibration

provides an analog voltage, proportional to the measured signal level expressed in $\mathrm{dBm}$. This analog value is filtered by a low pass filter and sampled by a 16-bit analog-to-digital converter, controlled via a serial programming (SPI) interface.

\section{D2 Processing the measurement}

The embedded software provides accurate timing of the measurement and calculates minimum, maximum, linear and geometric averages over one-second time intervals. The necessary conversions from $\mathrm{dBm}$ to power on a linear scale, and conversely, are performed by a table-based algorithm to optimize execution speed on the low-power micro controller. 


\section{Storing the results}

The processed results are expressed in $\mathrm{dBm}$ and stored in local flash memory at one-second time intervals. A check sum is included, for verification during data readout. The high clock accuracy synchronizes measurements over extended periods in time.

\section{D4 Recording time stamps}

Time stamps can be recorded at user-defined moments to mark the beginning of a specific measurement situation. This is performed by pressing a button on the portable master unit, which sends a broadcast time stamp packet over the system bus, triggering all active nodes to log the time stamp into their local flash memories.

\section{D5 Communication with the master unit}

All slave nodes communicate with the master unit, which has full control over the communication, through a custom system bus protocol using serial communication with 9 data bits. The ninth data bit signals whether the forwarded value is a node address or data. The master unit may trigger a specific slave node to forward the contents of its local flash memory on the bus, erase the flash memory, or inform about the status of the node for monitoring purposes during the measurement.

\section{MEASUREMENT RESULTS}

The measurement result displayed in Fig. 4 shows the power densities of signals impinging on the body for the GSM 900 and $1800 \mathrm{MHz}$ bands, uplink as well as downlink channels, for a city walk. The results shown in the graph are combined values, measured front and back of the body for each band. Although data is available for all 11 bands, a selection of 4 bands was made to obtain a clear graph. The system is successful in measuring the data synchronously and in discriminating the different bands. The downlink signals, originating from the base stations, are clearly the strongest in both bands. Uplink signals, from the GSM users, are generally weak, except when approaching an active user.

\section{CONCLUSION}

The measurement system developed for the ACCEDERA project, including 22 active on-body nodes is successfully used in an ongoing measurement campaign in multiple European countries. The system is worn in an unobtrusive way and has a large autonomy on commonly available battery power

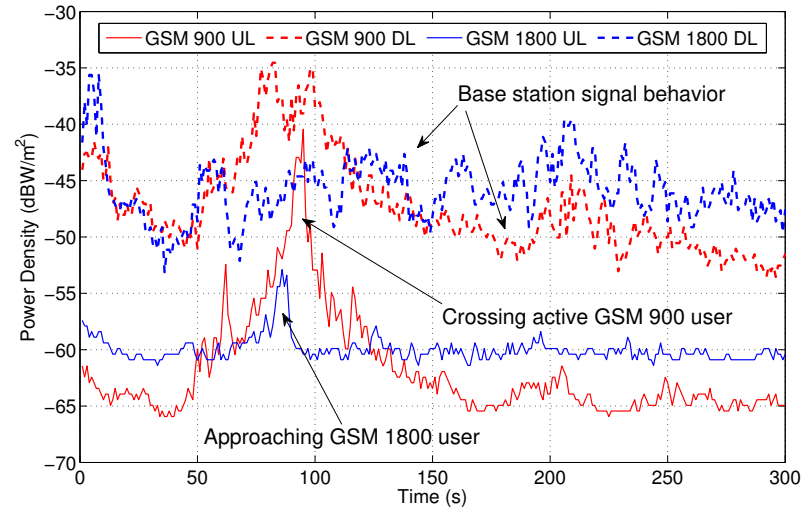

Fig. 4: Measurement for GSM 900 and $1800 \mathrm{MHz}$ uplink and downlink bands, for a city walk

packs. Measurements indicate accurate synchronous sampling of exposure at different frequency bands. The personal distributed exposimeter for 11 frequency bands, with front and back antennas for each band, provides a large amount of unique and valuable measurement data.

\section{ACKNOWLEDGEMENTS}

Sam Agneessens is a postdoctoral Fellow with the FWO-V. The authors would like to thank the French Agency for Food, Environmental and Occupational Health \& Safety (ANSES) for funding the project "Amélioration de la précision des mesures personnelles aux fréquences radio et caractérisation des niveaux d'exposition dans divers environnements et différents pays", ACCEDERA, 2015-2017.

\section{REFERENCES}

1. Thielens Arno, Agneessens Sam, Verloock Leen, et al. On-body calibration and processing for a combination of two radio-frequency personal exposimeters RADIATION PROTECTION DOSIMETRY. 2015;163:5869.

2. Thielens Arno, Vanveerdeghem Peter, Agneessens Sam, et al. Wholebody averaged specific absorption rate estimation using a personal, distributed exposimeter IEEE ANTENNAS AND WIRELESS PROPAGATION LETTERS. 2015;14:1534-1537.

3. Agneessens Sam, Rogier Hendrik. Compact half diamond dual-band textile HMSIW on-body antenna IEEE TRANSACTIONS ON ANTENNAS AND PROPAGATION. 2014;62:2374-2381.

4. Agneessens Sam, Lemey Sam, Vervust Thomas, Rogier Hendrik. Wearable, small, and robust: the circular quarter-mode textile antenna IEEE ANTENNAS AND WIRELESS PROPAGATION LETTERS. 2015;14:1482-1485.

5. Vanveerdeghem Peter, Van Torre Patrick, Thielens Arno, Knockaert Jos, Joseph Wout, Rogier Hendrik. Compact personal distributed wearable exposimeter IEEE SENSORS JOURNAL. 2015;15:4393-4401. 